\title{
MANIFESTAÇÃO ORAL DA LEUCEMIA MIELÓIDE AGUDA COMO PRIMEIRO SINAL PARA O DIAGNÓSTICO \\ - relato de caso •
}

\author{
Dhiancarlo Rocha Macedo ${ }^{a}$ \\ Carlos Henrique Alves de Rezende ${ }^{b}$ \\ Rogério Moreira Arcieri ${ }^{\mathrm{C}}$ \\ Renata Silva Barbosa ${ }^{d}$
}

\section{Resumo}

\begin{abstract}
A leucemia mieloide aguda (LMA) é uma doença maligna da medula óssea que pode apresentar manifestações sistêmicas e bucais. O objetivo deste trabalho é descrever um caso clínico de um paciente, 16 anos de idade, que apresentou como manifestação bucal lesões ulceradas em dorso de língua e linfadenopatia em região cervical à direita. Após exame clínico e solicitação de exames complementares, teve-se como hipótese o diagnóstico de leucemia aguda, sendo o paciente encaminhado ao serviço médico especializado. Este trabalho mostra a importância do cirurgião-dentista no diagnóstico inicial da leucemia.
\end{abstract}

Palavras-chave: Leucemia mieloide aguda; Manifestações bucais; Diagnóstico precoce.

\footnotetext{
a. Cirurgião-Dentista do Hospital do Câncer de Uberlândia e Hospital Odontológico da UFU. Precepto rda Residência Multiprofissional com atenção em Oncologia do Hospital de Clíncas da UFU. dentistamacedo@hotmail.com

b. Mestre e Doutor em Medicina. Professor Associado da Faculdade de Medicina da UFU. charezende@ufu.br

c. Mestre e Doutor em Odontologia Preventiva e Social. Professor Titular da Faculdade de Odontologia da Universidade federal de Uberlândia.ops@umuarama.ufu.br

d. Estudante do curso de Graduação em Odontologia do Centro Universitário do Triângulo - UNITRI. renataquiri@hotmail.com
} 


\title{
ORAL EXPRESSION ACUTE MYELOID LEUKEMIA AS FIRST SIGN FOR DIAGNOSIS
}

\author{
- case report•
}

\begin{abstract}
Acute myeloid leukemia ( $A M L)$ is a malignant disease of the bone marrow that can present systemic and oral manifestation. In this case report we describe a patient, 16 years of age, who presented the oral manifestation ulcerated lesions on the dorsum of the tongue and cervical lymph node in the right region. After clinical examination and complementary tests, it was taken as a hypothesis the diagnosis of acute leukemia, and the patient was referred to a specialized medical service. Showing the importance of the dentist in the initial diagnosis of leukemia.
\end{abstract}

Keywords: Acute myeloid leukemia; Oral manifestation;

Early diagnosis.

\section{INTRODUÇÃO}

As leucemias são neoplasias malignas hematológicas caracterizadas pela proliferação desregulada de células progenitoras imaturas da hematopoese, sendo originadas na medula óssea e, com a sua evolução, atingem a corrente sanguínea e outros órgãos como baço, fígado, linfonodos, pele, cavidade oral, sistema nervoso e outros tecidos em todo o corpo. ${ }^{(1,9,10)}$

A leucemia pode ser classificada em aguda e crônica, de acordo com o seu comportamento clínico e caracterizada como mieloide e linfoide, de acordo com a sua origem histogenética. ${ }^{(2)}$ As leucemias agudas são marcadas pelo avanço de células cancerosas imaturas na medula óssea e no sangue periférico. (3) $O$ tratamento da leucemia mieloide aguda (LMA) inclui quimioterapia, sendo uma fase de indução da remissão, seguida consolidação da remissão e uma fase de manutenção quimioterápica. O uso do Transplante de Medula Óssea (TMO) estaria reservado aos casos de recidiva ou àqueles pacientes portadores de pior prognóstico. ${ }^{(8)}$

Os sinais e sintomas da leucemia aguda são decorrentes do acúmulo e da proliferação de leucócitos alterados na medula óssea, prejudicando ou impedindo a produção de glóbulos vermelhos (causando anemia, cansaço e palidez das mucosas ou pele), glóbulos brancos (causando neutropenia e aumentando o risco de infecções) e plaquetas (causando trombocitopenia e maior risco de hemorragia). ${ }^{(1,4)} \mathrm{O}$ hemograma está quase sempre alterado. Dentre as alterações laboratoriais mais observadas estão à anemia, a trombocitopenia e a presença de blastos na contagem diferencial de leucócitos. ${ }^{(5,10)}$ 
Dentre as manifestações bucais do paciente com leucemia aguda pode ocorrer a presença de equimoses, petéquias e sangramento gengival espontâneo devido à trombocitopenia. ${ }^{(1,9,10)} \mathrm{A}$ infiltração gengival por células leucêmicas também podem ocorrer, levando a um aumento do volume gengival. ${ }^{(6,10)}$ Além da neutropenia que pode aumentar a susceptibilidade à infecções fúngicas, bacterianas e viróticas. ${ }^{(5)}$ As manifestações bucais podem estar presentes em até $89 \%$ dos estágios iniciais da leucemia, o que demonstra a importância do cirurgião-dentista no diagnóstico precoce das leucemias agudas. ${ }^{(7)}$

Este trabalho tem o objetivo de relatar o caso de um jovem com leucemia mieloide aguda que apresentou manifestação bucal em língua e linfonodos cervicais, decorrente da doença.

\section{CASO CLÍNICO}

Paciente, sexo masculino, 16 anos, leucoderma, procurou o Pronto Socorro Odontológico do Hospital de Clínicas da Universidade Federal de Uberlândia acompanhado de seu pai, queixando-se de ferida em língua e odinofagia.

Durante a anamnese o paciente relatou que há quinze dias apresentou dor de garganta, febre baixa, desânimo e fraqueza e procurou o serviço médico no qual foi prescrito a ele amoxicilina $500 \mathrm{mg}$ de 08 em 08 horas por sete dias, pois o médico havia suspeitado de uma possível amigdalite, sem haver melhora da sintomatologia. Cerca de cinco dias após finalizar o uso do antibiótico o paciente notou o surgimento de feridas no dorso lingual.

No exame clínico da cavidade oral foi possível observar o dorso lingual recoberto de lesões avermelhadas com aspecto ulcerado e endurecido à palpação (figura 1) e também linfadenopatia, dolorosa à palpação, em região cervical do lado direito (figura 2), o restante da mucosa oral apresentava-se com aspecto de normalidade. Em um primeiro momento, devido à idade do paciente e localização da lesão em mucosa queratinizada, foi estabelecida como hipótese de diagnóstico a manifestação primária do herpes simples ou mononucleose infecciosa. Mas devido aspecto petroso da língua e sintomas sistêmicos relatados, optou-se por solicitar um hemograma de urgência, pois se cogitou a hipótese de uma possível infiltração de células malignas, além dos exames de sorologia (Anti-HIV, Monotest, Anti-CMV e VDRL).

O resultado dos exames de sorologia foram todos negativos, enquanto o hemograma evidenciou um quadro de leucocitose $(165 \mathrm{mil} / \mathrm{mm} 3)$, anemia e presença de blastos na contagem diferencial de leucócitos (figura 3), sendo o paciente encaminhado imediatamente ao Hospital do Câncer de Uberlândia com suspeita de leucemia aguda. Nenhum tratamento específico foi realizado, pela equipe de odontologia, para as ulcerações da língua, pois confirmando o diagnóstico da leucemia, a própria quimioterapia levaria há uma regressão da lesão oral.

No serviço de oncologia foi realizado o mielograma e a imunofenotipagem tendo como diagnóstico definitivo Leucemia mieloide aguda tipo $\mathrm{M}_{5}$.

Em seguida o paciente iniciou o tratamento quimioterápico, sendo utilizados como base os quimioterápicos citarabina, etoposide e doxorrubicina. O ciclo de quimioterapia era mensal. Após o primeiro ciclo de quimioterapia houve uma alteração clínica da língua, mas sem regressão da lesão (figura 4). A completa regressão da lesão em língua ocorreu após o segundo ciclo de quimioterapia (figura 5). Após quatro ciclos de quimioterapia, o paciente realizou o transplante de medula óssea alogênico, no Hospital de Clínicas da Universidade Federal de Minas Gerais - UFMG, na qual a doadora foi sua irmã gêmea. Atualmente, o paciente encontra-se em acompanhamento médico no mesmo hospital. 


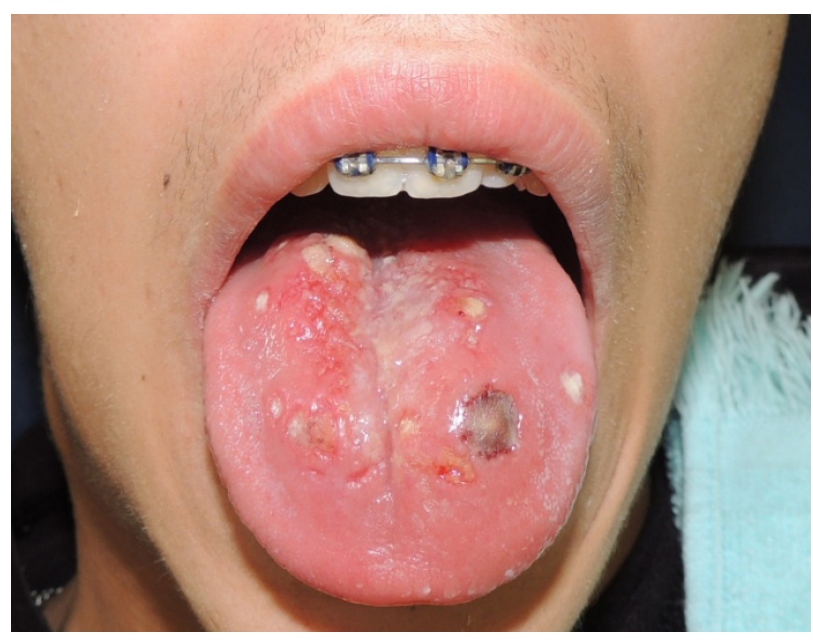

Figura 1 - Aspecto ulcerado e eritematoso em dorso lingual

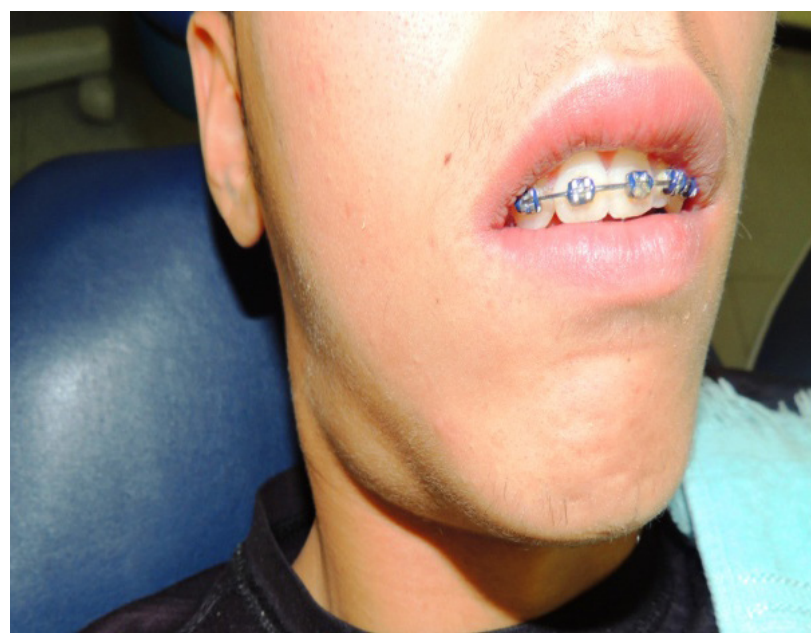

Figura 2 - Linfadenopatia em região cervical à direita

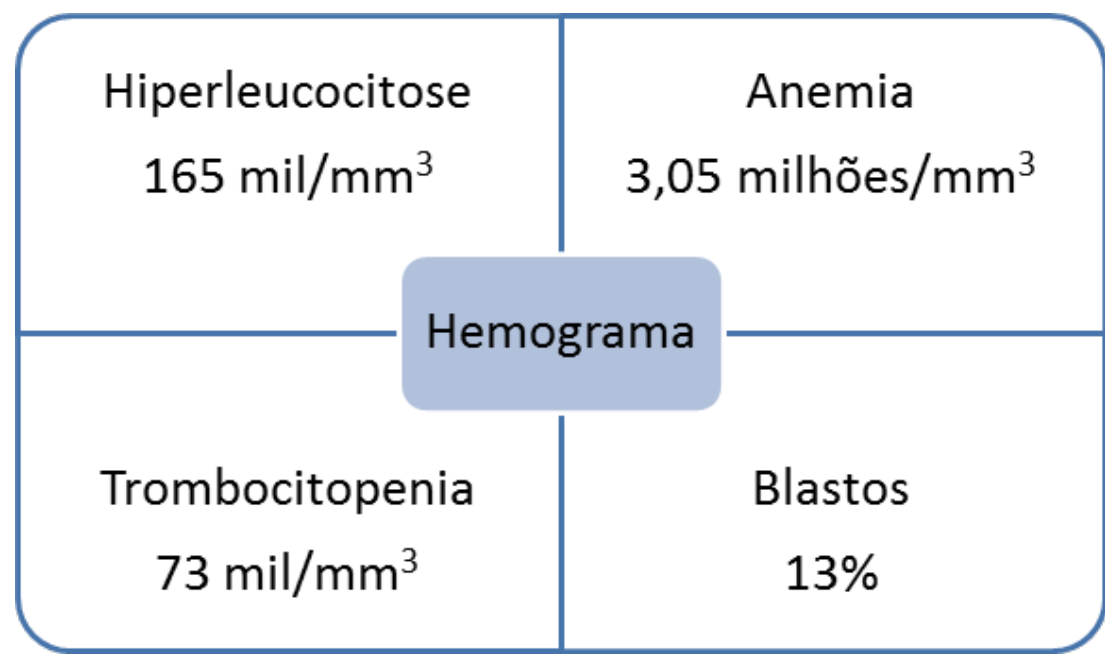

Figura 3 - Hemograma evidenciando leucocitose, trombocitopenia, anemia e presença de blastos.

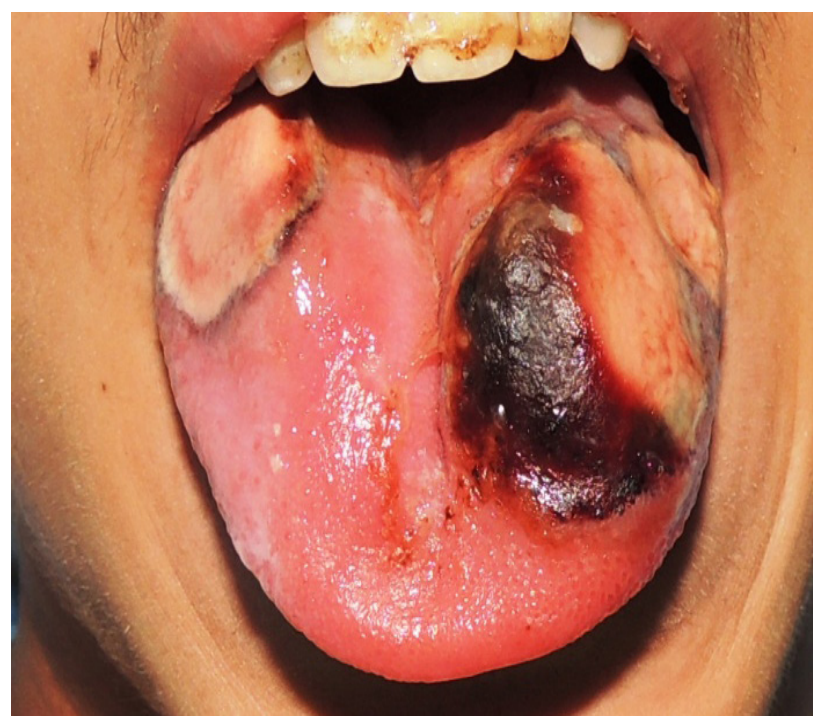

Figura 4 - Aspecto do dorso lingual após 10 ciclo de quimioterapia. Nota-se que o aparelho ortodôntico foi removido.

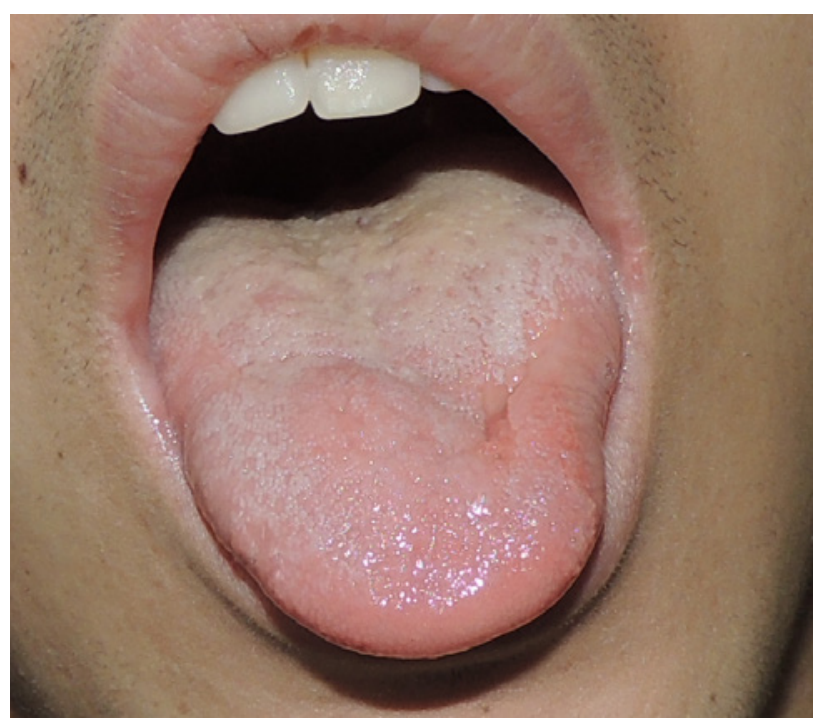

Figura 5 - Regressão da lesão após 20 ciclo de quimioterapia 


\section{DISCUSSÃO}

A gengiva é o local mais comum de infiltração de células leucêmicas em cavidade oral, levando a um aumento do volume gengival e sangramento. ${ }^{(1,2,6,10)}$ E neste caso apresentado, a infiltração das células leucêmicas ocorreu na língua, o que levou a cogitarem-se outras hipóteses de diagnóstico.

Dentre estas hipóteses estavam a manifestação primária do herpes simples e a mononucleose infecciosa, devido a estas patologias apresentarem sintomas e sinais clínicos semelhantes ao relatado pelo paciente como odinofagia, febre, enfartamento ganglionar e ulcerações em mucosa queratinizada. ${ }^{(11,12,13,14)}$

Pacientes leucêmicos podem apresentar sintomas sistêmicos como cansaço, fraqueza, desânimo, decorrentes principalmente da anemia, além de manifestações bucais como petéquias, equimoses e sangramento gengival devido ao quadro de trombocitopenia. (1, 4, 9,10) Dentre estes sinais e sintomas relatados na literatura, o paciente relatou durante anamnese desânimo e cansaço e em relação à cavidade oral apresentou apenas as ulcerações em dorso lingual, estando as demais mucosas íntegras.

Em relação aos exames complementares, que podem auxiliar no diagnóstico das leucemias agudas, está o hemograma, que quase sempre se encontra alterado apresentando hiperleucocitose, anemia, trombocitopenia, neutropenia e presença de blastos na contagem diferencial de leucócitos. $\left.{ }^{5}, 10\right)$ No hemograma solicitado ao paciente tiveram-se como resultado uma leucocitose no valor de $165 \mathrm{mil} / \mathrm{mm}^{3}$, anemia, trombocitopenia (75 mil/ $\mathrm{mm}^{3}$ ) e presença de blastos (13\%), não apresentando apenas neutropenia. Sendo este quadro compatível com uma provável leucemia aguda.

Para o diagnóstico definitivo da leucemia é necessário a realização do mielograma. Este exame é feito sob anestesia local e consiste na aspiração da medula óssea seguida da confecção de esfregaços em lâminas de vidro, para exame ao microscópio. Os locais preferidos para a aspiração são a parte posterior do osso ilíaco e o esterno. ${ }^{(8)} \mathrm{O}$ paciente realizou este exame assim que entrou no serviço de oncologia do Hospital do Câncer de Uberlândia, confirmando o diagnóstico de leucemia mieloide aguda.
Já a imunofenotipagem detecta e identifica os marcadores celulares expressos em cada tipo e subtipo das leucemias agudas, mostrando em que estágio de desenvolvimento encontra-se o clone leucêmico. ${ }^{(8)}$ Este exame também realizado pelo paciente é que classificou a sua LMA no subtipo M5.

Este caso mostra a grande importância do cirurgião-dentista no diagnóstico de uma provável leucemia, pois $89 \%$ das leucemias em estágio inicial podem apresentar manifestação bucal. (7)

\section{CONSIDERAÇÕES FINAIS}

A leucemia pode ter a suas primeiras manifestações na mucosa oral, o que reafirma a importância do cirurgião-dentista no diagnóstico precoce da doença. E este profissional deve estar atento e preparado para reconhecer qualquer tipo de alteração, seja de ordem geral e principalmente bucal de seus pacientes.

Sendo a hipótese de diagnóstico sugestiva de uma patologia sistêmica, como por exemplo, uma neoplasia maligna, obrigatoriamente deve-se encaminhar o paciente a um serviço médico especializado.

\section{REFERENNCIAS}

1. Menezes L, Rao JR. Acute myelomonocytic leukemia presenting with gingival enlargement as the only clinical manifestation. Journal of Indian Society of Periodontology. 2012; 16(4): 597-601.

2. Demirer S, Ozdemir H, Sencan M, Marakoglu I. Gingival hyperplasia as an early diagnostic oral manifestation in acute monocytic leukemia: a case report. European journal of dentistry. 2007; 1(2): 111-4.

3. Visacre PHM, Saez CRN, Oliveira AV. O transplante Autólogo como forma de tratamento da leucemia. Saúde e Pesquisa. 2011; 4(2): 289-298.

4. Santos VI, Anbinder AL, Cavalcante ASR. Leucemia no paciente pediátrico: atuação odontológica. Brazilian Dental Science. 2010; 6(2): 49-57. 
5. Carneiro FM, Silva LCP, Cruz RA.

Manifestações bucais das leucemias agudas na infância. Arquivo Brasileiro de Odontologia. 2010; 4(1): 40-54.

6. Santos PSS, Silva BSF, Coracin FL, Yamamoto FP, Junior DSP, Magalhães MG. Granulocytic sarcoma of the oral cavity in a chronic myeloid leukemia patient: An unusual presentation. Med Oral Patol Oral Cir Bucal. 2010 Mar; 15(2): e350-2.

7. Andrade FA, Santos PSS; Freitas RR. Manifestações bucais em pacientes com leucemia mielóide aguda (LMA). Arq Med Hosp Fac Cienc Med Santa Casa São Paulo. 2008; 53(2): 85-7.

8. Ministério da Saúde. Instituto Nacional do Câncer. Rio de Janeiro: INCA; 2O14. Disponível em HTTP://www.inca.gov.br. Acesso em: 16 jun. 2014.

9. Fatahzadeh M, Krakow AM. Manifestation of acute monocytic leukemia in the oral cavity: a case report. Spec Care Dentist. 2008; 28(5): 190-94.
10. Santos PSS, Fontes A, Andrade F, Sousa SCOM. Gengival leukemic infiltration as the first manifestation of acute myeloid leukemia. Otolaryngology - Head and Neck Surgery. 2010; 143: 465-66.

11. Neville BW, Damn DD, Allen CM, Bouquot JE. Patologia Oral e Maxilofacial. 2. ed. Rio de Janeiro: Guanabara Koogan; 2004.

12. Santos PSS, Silva BSF, Coracin FL, Yamamoto FP, Junior DSP, Magalhães MG. Granulocytic sarcoma of the oral cavity in a chronic myeloid leukemia patient: An unusual presentation. Med Oral Patol Oral Cir Bucal. 2010 Mar; 15(2): e350-2.

13. Andrade FA, Santos PSS; Freitas RR. Manifestações bucais em pacientes com leucemia mielóide aguda (LMA). Arq Med Hosp Fac Cienc Med Santa Casa São Paulo. 2008; 53(2): 85-7.

14. Ministério da Saúde. Instituto Nacional do Câncer. Rio de Janeiro: INCA; 2O14. Disponível em HTTP://www.inca.gov.br. Acesso em: 16 jun. 2014. 